\title{
Telangiectasia retiniana justafoveolar idiopática do 2A: fiperpigmentaçãa macular incomum em dois do grupo pacientes
}

Idiopathic juxtafoveolar retinal telangiectasis group $2 \mathcal{A}$ : an unusual macular
hyperpigmentation in two patients

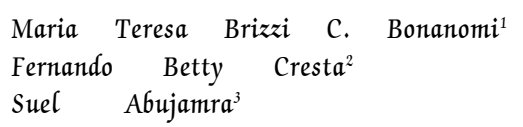

${ }^{1}$ Doutora em Oftalmologia, Médica Assistente da Divisão de Retina da disciplina de Oftalmologia da Faculdade de Medicina da Universidade de São Paulo.

${ }^{2}$ Médico Assistente do Hospital Universitário da Universidade de São Paulo e Pós-Graduando do Hospital das Clínicas da Universidade de São Paulo

${ }^{3}$ Professor Associado da Disciplina de Oftalmologia da Faculdade de Medicina da Universidade de São Paulo, Professor Titular de Oftalmologia da Faculdade de Medicina de Santo Amaro.

Endereço para correspondência: Rua Professor João de Oliveira Torres, 93 - São Paulo (SP) CEP 03337-010. E-mail: fbcresta@yahoo.com

\section{RESUMO}

Introdução: Telangiectasias retinianas justafoveolares idiopáticas (TJI) do grupo $2 \mathrm{~A}$ ocorrem na $5^{\text {a }}$ ou $6^{\text {a }}$ década de vida, em ambos os sexos. A diminuição da visão está associada a alterações características na mácula que incluem depósitos de pigmento. Objetivo: Descrever dois pacientes com achados incomuns de pigmentação macular. Método: Relato de caso e discussão. Resultados: Observação de grandes placas de pigmento na mácula de dois pacientes. Nos dois casos houve confusão diagnóstica com coriorretinite macular cicatrizada. Em um caso demonstrou-se o aumento da quantidade de pigmento em um período de oito anos. Conclusão: A TJI do grupo $2 \mathrm{~A}$ pode ser de diagnóstico difícil. A suspeita clínica deve ser feita pelo aspecto característico das placas de pigmento na mácula.

Descritores: Telangiectasia/diagnóstico; Fóvea central/patologia; Mácula lútea/patologia; Epitélio pigmentado ocular; Neovascularizaçao retiniana; Angiofluoresceinografia; Fundo de olho; Diagnóstico diferencial; Relato de caso

INTRODUÇÃO

Telangiectasia retiniana foi o termo proposto por Reese para descrever um grupo de retinopatias caracterizadas por vasos dilatados e incompetentes $^{(1)}$. Quando situadas apenas na região macular, as telangiectasias são ditas justafoveolares. O termo telangiectasia justafoveolar idiopática (TJI) foi introduzido por Gass \& Oyakawa em 1982(2). Atualmente usa-se a classificação aperfeiçoada por Gass \& Blodi em 1992 onde, o grupo 1 que é uma variante da síndrome de Coats e os grupos 2 e 3 são afecções $\operatorname{adquiridas}^{(3)}$.

O subgrupo 2A é o tipo mais freqüente das TJI, apresentando-se com diminuição da visão bilateral na quinta ou sexta década de vida, com leve assimetria e afetando ambos os sexos. O exame ocular revela apenas alterações retinianas maculares que incluem: cor acinzentada da retina macular, depósitos cristalinos superficiais, veias em ângulo reto, placas de hiperpigmentação retiniana e neovascularização sub-retiniana ${ }^{(2-4)}$. As telangiectasias são visíveis apenas à angiofluoresceinografia. Estas alterações tendem a aparecer ou tornam-se mais evidentes com o passar do tempo ${ }^{(5)}$.

$\mathrm{Na}$ maioria dos casos a hiperpigmentação retiniana forma depósitos granulares finos na região macular ${ }^{(3)}$. Neste trabalho relatamos o achado incomum de dois pacientes que apresentavam grandes placas de pigmento. 
RELATO DE CASO

\section{Caso 1:}

P.T.B.S., 57 anos, sexo masculino, branco, advogado. Apresentou-se em 05/02/1989 para esclarecimento diagnóstico com queixa de diminuição gradativa da visão há 1 ano e suspeita de coriorretinite por toxoplasma. Os exames laboratoriais (VHS, fator reumatóide, ASLO e glicemia de jejum) apresentavam-se dentro da normalidade e a sorologia para toxoplasmose apresentava padrão de infecção pregressa. Os antecedentes individuais e familiares eram irrelevantes. Visão do olho direito (OD): $20 / 30\left(+0,75 \mathrm{DE}=-1,00 \mathrm{DC}\right.$ a $\left.90^{\circ}\right)$ e do olho esquerdo (OE): 20/60 (+0,50DE $=-0,50 \mathrm{DC}$ a $\left.80^{\circ}\right)$. Biomicroscopia normal em ambos os olhos (AO) e pressão intra-ocular de $16 \mathrm{mmHg}$ em AO. Ao exame de fundo de olho verificou-se cor acinzentada da retina perifoveolar, depósitos cristalinos e placas de pigmento na região temporal (Figura 1). A angiofluores-
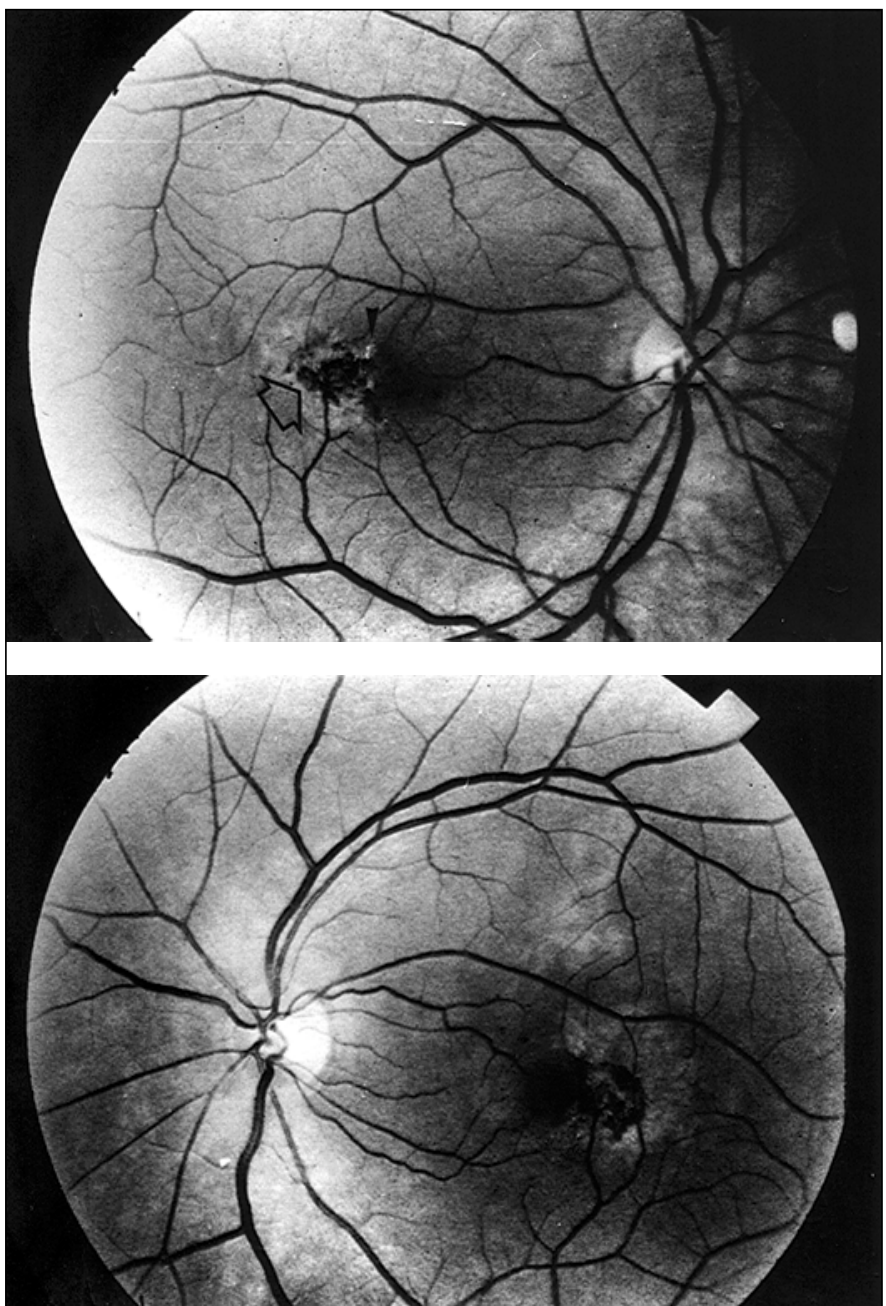

Figura 1 - Retinografia aneritra do paciente 1 em 1989. Placas pigmentares apenas na região temporal da mácula de $\mathrm{AO}$ com depósitos cristalinos (respectivamente seta e ponta de seta) ceinografia mostrou hipofluorescência por bloqueio causado pelo pigmento e hiperfluorescência das telangiectasias na metade temporal da mácula durante as fases de trânsito. $\mathrm{Na}$ fase de recirculação, toda a mácula apresentou hiperfluorescência borrada, indicando extravasamento do corante. Com o diagnóstico de TJI do grupo $2 \mathrm{~A}$ e conduta expectante, retornou em 02/10/1996 queixando-se de piora da AV, especialmente à leitura. Ao exame apresentava visão de 20/60 no OD e de 20/200 no OE, com aumento da quantidade de pigmento depositado, veias em ângulo reto e depósitos cristalinos na mácula de $\mathrm{AO}$ (Figura 2).

\section{Caso 2:}

M.L.R.P., 62 anos, sexo feminino, branca, do lar. Apresentou-se em 10/03/1999 dizendo ser portadora de visão subnormal por uma lesão no fundo de olho, provavelmente adquirida durante a infância ou mesmo antes do nascimento. Fazia uso de óculos de +9,00DE em AO para visão subnormal.
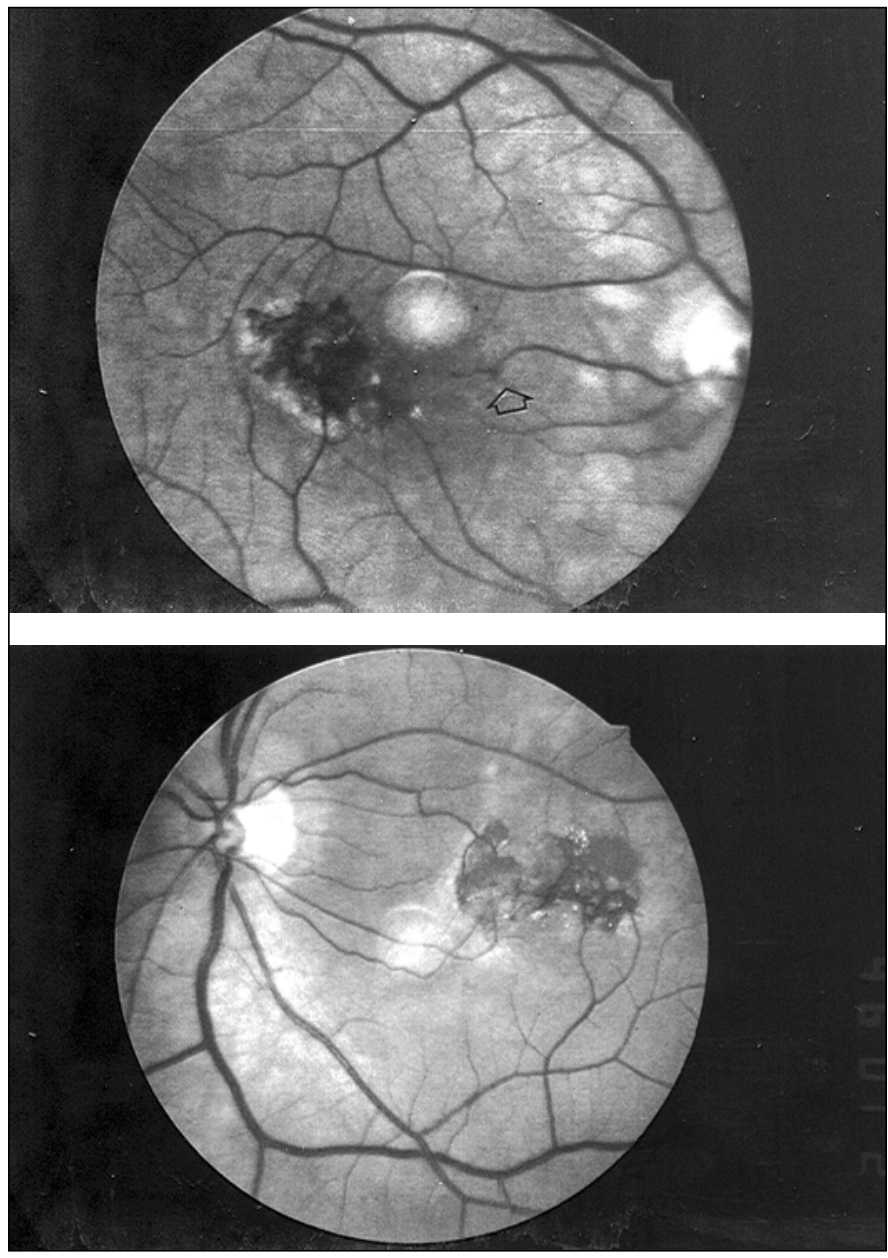

Figura 2 - Retinografia do paciente 1 em 1996. O OD apresenta placas espiculadas de pigmento de 1 diâmetro de disco na mácula temporal e depósito de pigmento puntiforme nasal (seta). O OE mostra placas de pigmento nas regiões nasal e temporal da mácula, veias em ângulo reto e depósitos cristalinos 
Hipertensa e portadora de osteoporose fazia uso de $\beta$-bloqueador e cálcio com reposição hormonal; os antecedentes familiares eram irrelevantes. Ao exame apresentava visão - OD: 20/200 (+2,50DE), OE: 20/80 (+2,50DE), biomicroscopia normal em AO e pressão ocular de $18 \mathrm{mmHg}$ em AO. O fundo de olho era semelhante em AO com leve assimetria sendo mais alterado no OD. O exame da mácula mostrava a retina espessada na região perifoveolar, veias em ângulo reto, depósitos cristalinos superficiais e placas de pigmento de forma estrelada, coalescentes, na região temporal e nasal da fóvea, ligados por istmos de pigmento (Figura 3). O angiograma mostrava hipofluorescência por bloqueio causado pelo pigmento e hiperfluorescência tardia com extravasamento do corante na região macular (Figura 3). Com o diagnóstico de TJI do grupo $2 \mathrm{~A}$ foi indicada a conduta expectante.
DISCUSSÃO

A TJI do grupo 2A é uma vasculopatia de causa desconhecida e considerada uma afecção adquirida dos capilares profundos da mácula. Os achados de microscopia óptica e eletrônica mostram um espessamento da parede e não aumento da luz dos capilares $^{(5)}$. Gass e Blodi ${ }^{(3)}$ sugerem uma seqüência no aparecimento das alterações anatômicas da mácula destes pacientes, classificando-a em 5 estágios: Estágio 1, sem alterações biomicroscópicas, com hiperfluorescência tardia na região temporal da mácula ao angiograma; Estágio 2, coloração acinzentada da mácula perifoveolar, aparecimento das telangiectasias durante a fase de trânsito e extravasamento do corante nas fases tardias do angiograma; Estágio 3, aparecimento das veias em ângulo reto que drenam


Figura 3 - Paciente 2. As fotos aneritras (superiores) mostram o OD com placas de pigmento nas regiões nasal e temporal da mácula, depósitos cristalinos (seta) e veias em ângulo reto (ponta de seta) e o OE com pigmento sobre a fóvea. $\mathrm{Na}$ angiofluoresceínografia (fotos inferiores) vê-se bem $o$ istmo ligando as placas de pigmento (seta) 
a rede profunda de capilares, inicialmente na região temporal da mácula; Estágio 4; placas de pigmento de formato estrelado situadas, em geral, junto à emergência das veias em ângulo reto; Estágio 5, neovascularização sub-retiniana e anastomose corio-retiniana. Os depósitos cristalinos, considerados produtos da degeneração das células de Müller $^{(2)}$, são comuns ${ }^{(3-4,6)}$ e mais evidentes a partir do estágio 3.

A TJI do grupo 2 pode ser de difícil diagnóstico, especialmente nos estágios iniciais. A presença da placa pigmentar facilita a lembrança do diagnóstico. Elas são em geral temporais, de formato estrelado e associadas a uma veia em ângulo reto (Figura 1). Quando a quantidade de pigmento aumenta, como foi demonstrado no caso 1, o quadro fundoscópico pode ser confundido com o de outra maculopatia. A confusão diagnóstica mais comum é com cicatriz macular por coriorretinite, como foi pensado nos dois casos apresentados. Na cicatriz macular há atrofia da retina com hiperplasia do epitélio pigmentado enquanto que na TJI do grupo 2 há aumento de espessura da retina macular ao exame biomicroscópico. As retinografias apresentadas mostram que as placas pigmentares são diferentes dos casos de cicatriz corioretiniana. Além do formato estrelado já descrito, apresentam uma distribuição em dois agrupamentos, um nasal e outro temporal, poupando a área que corresponde a zona avascular da fóvea. Esta localização está bem evidente no OE do caso 1 (Figura 2) e no OD do caso 2 (Figura 3). Nota-se que entre as duas porções de pigmento, esboça-se um istmo de união. Considerando-se a origem da doença nos capilares profundos da mácula (5) e a fisiopatologia proposta (3), esta localização anatômica, perifoveolar, seria esperada. Apenas no OE do paciente 2 há depósito sobre a fóvea (Figura 3).

A outra alteração que faz pensar neste diagnóstico são os depósitos cristalinos, que estão presentes em todas as retinografias apresentadas. Sua localização bem superficial, ao nível da membrana limitante interna e sua dimensão punctiforme e distribuição justa ou perifoveolar são características.

Apesar da ausência de pigmento sobre a fóvea pode haver diminuição da acuidade visual nestes pacientes. Considera-se que o comprometimento da visão ocorra pela degeneração dos fotorreceptores, conseqüente às alterações anatômicas crônicas e progressivas na região macular ${ }^{(3,7)}$.

Em conclusão, analisamos o padrão de hiperpigmentação macular da TJI do grupo 2 em dois pacientes. Em um deles pôde ser demonstrado o aumento da quantidade de pigmento em um período de 8 anos. Ressaltamos a importância das características das placas pigmentares e da presença dos depósitos cristalinos para o diagnóstico desta maculopatia.

\section{A B S T R ACT}

Background: Idiopathic juxtafoveolar retinal telangiectasis (IJT) group 2A occurs in the $5^{\text {th }}$ or $6^{\text {th }}$ decade of life and affects both sexes. The visual loss is associated with characteristic fundus findings that include pigment deposition in the macula. Purpose: To describe two cases with uncommon macular pigmentation. Method: Case report and discussion. Results: We demonstrated heavy pigment deposition in the macula of two patients who were previously misdiagnosed as having macular corioretinal scar. Pigment deposition enhancement was shown in the follow-up period of eight years in one patient. Conclusion: Group 2A IJT diagnosis may be difficult and should be considered based on the clinical features of the pigment deposits in the macula.

Keywords: Telangiectasis/diagnosis; Fovea centralis/pathology; Macula lutea/pathology; Pigment epithelium of eye; Retinal neovascularization; Fluorescein angiography; Fundus oculi; Differential diagnosis; Case report

\section{REFERÊNCIAS}

1. Reese AB. Telangiectasia of the retina and Coats'disease. Am J Ophthalmol $1956 ; 42: 1-8 ?$

2. Gass JD, Oyakawa RT. Idiopathic juxtafoveal retinal telangiectasis. Arch Ophthalmol 1982;100:769-80.

3. Gass JD, Blodi BA. Idiopathic juxtafoveolar retinal telangiectasis. Update of classification and follow-up study. Ophthalmology 1992;100:1536-46.

4. Abujamra S, Bonanomi MTBC, Machado CG, Cresta FB, Pimentel SLG, Caramelli CB. Telangiectasia retiniana justafoveolar idiopática: padrão clínico e fundoscópico em 19 casos. Rev Bras Oftalmol 1998;57:481-7.

5. Green WR, Quigley HA, De la Cruz Z, Cohen B. Parafoveal retinal telangiectasis. Light and electron microscopy studies. Trans Ophthalmol Soc U.K. 1980(Pt1);100:162-70.

6. Moisseiev J, Lewis H, Bartov E, Fine SL, Murphy R. Superficial retinal refractile deposits in juxtafoveal telangiectasis. Am J Ophthalmol 1990;109: 604-5.

7. Park D, Schatz H, McDonald HR, Johnson RN. Fibrovascular tissue in bilateral juxtafoveal telangiectasis. Arch Ophthalmol 1996;114:1092-6.

\title{
ABO ELETRÔNICO
}

\author{
Novo site
}

Acesso:

fittp://www.abonet.com.br 